\title{
Dissipative Systems: From Physics to Robust Nonlinear Control
}

\author{
Antonio A. Alonso*, Carlos V. Fernandez, Julio R. Banga
}

March 21, 2003

\begin{abstract}
In this paper we explore connections between the underlying physics of dissipative systems and nonlinear robust control. In particular, we concentrate on the problem of stabilizing stationary solutions of nonlinear dissipative systems with states distributed in space. Dissipative systems are equipped with an entropy function which we employ to relate dissipation with the Hamilton-Jacobi-Bellman equation. This relation allows us to establish formal links between the dynamic properties of dissipative systems, passivity and optimal stabilizing control, as it is understood in systems theory. Robustness issues in controller design, are also discussed in the context of front or pulse spatial pattern stabilization.
\end{abstract}

Process Engineering Group, IIM-CSIC, Eduardo Cabello 6, 36208 Vigo, Spain

* Corresponding author antonio@iim.csic.es

Keywords: Dissipative Systems, Distributed Process Systems, Passivity, Hamilton-JacobiBellman, Optimal Control, Minimum Entropy Production, Robust Control 


\section{Introduction}

Dissipation is a physical concept closely related to the first and the second law of thermodynamics. The first law ensures conservation of mass and energy in all its forms. The second law determines the way in which the different forms of energy and material species evolve (relate and convert one into each other) through transport phenomena and chemical reactions, taking place on a given spatial domain. The evolution criterion is formally stated in terms of a concave function, called entropy, which never decreases in isolated processes and achieves its maximum at equilibrium. Thus, systems out of equilibrium spontaneously evolve to equilibrium through irreversible processes that produce entropy. In this way, dissipation is a positive function that quantifies the rate at which entropy is produced (Glansdorff and Prigogine, 1971).

This notion remains valid for open systems, we will refer to as Dissipative Systems. Now, in addition to entropy production, there exists an entropy flux between the system and its surroundings as materials and energy flow through the domain. In this context, dissipation imposes a particular relationship between transport processes and their associated thermodynamic forces (gradients) which guides the dynamic evolution of the system. However, the combined action of fluxes and rate processes can move the states of the system far away from equilibrium thus giving room to a rich variety of complex behaviors. From a control perspective, understanding the interplay between fluxes and dissipation seems essential to guide (control) the evolution of dissipative systems. Such objective was stated in 1934 by Donnan and Guggenheim (Demirel, 2002) in the following terms:

"A finite amount of organization may be purchased at the expense of a greater amount of disorganization in a series of interrelated spontaneous actions"

With the intention of developing efficient ways of purchasing organization, we explore, in this paper, connections between the underlying physics of dissipative systems and nonlinear robust control. In particular, we concentrate on the problem of stabilizing stationary solutions of nonlinear dissipative systems with states distributed in space (Distributed Process Systems). This class of dissipative systems plays a central role in many biological systems (Murray, 1993; Demirel, 2002) as well as in chemical and material processing industries (Christofides and Daoutidis, 1997a), as many of its operations involve convection diffusion and reaction phenomena. Interesting examples include, to name a few, catalytic reactors, chemical vapor deposition units, crystallization or thermal processing.

The control of distributed process systems has received considerable attention from the control community over the last years. Excellent surveys on this topic, covering both theoretical and applications aspects, can be found in (Balas, 1983; Lasiecka, 1995; Christofides, 2001). Standard approaches rely on a state-space-like representation of the original infinite dimensional system by spatial discretization of the set of partial differential equations. Common discretization schemes include finite differences or finite elements. Standard linear or nonlinear finite dimensional control design methods are then employed to construct the controller (see for instance Dochain et al, 1992). 
Alternative control design methods, which take into account the spatially distributed nature of the system, are based on spectral decomposition schemes which retains the essential properties of the spatial differential operator. This approach was extensively employed by Christofides and co-workers to derive robust stabilizing nonlinear controllers based on feed-back linearization (Christofides and Daoutidis, 1996; Christofides and Daoutidis, 1997b; Christofides, 2001).

A different -although complementary- approach is the one proposed by Ydstie and Alonso (1997) and Alonso and Ydstie $(1996 ; 2001)$ to develop passive stabilizing controls for distributed process systems. The approach settles its roots on the second law of thermodynamics and passivity, as it is understood in systems theory (Desoer and Vidyasagar, 1975; Sepulchre et al, 1997). The second law, in the exergy form, gives convexity which in turns provides a general answer to the question of finding Lyapunov function candidates to assess system's evolution. Passivity concepts link inputs to outputs while preserving the infinite dimensional structure of the system. These two concepts were employed by Hangos et al (1999) to assess structural stability properties in chemical process plants. Alonso et al (2000) applied them to design stabilizing high gain decentralized controllers for convection-diffusion-reaction processes. The theory was recently extended to hyperbolic process systems by Ydstie (2002).

In this work, we maintain the thermodynamic formalism to explore new links between the underlying physics of dissipative process systems and nonlinear control. The existence of an entropy-like function will allow us to relate dissipation with a Hamilton-Jacobi-Bellman type equation. Such connection will open direct ways to establish passivity conditions for dissipative systems. In this regard, one main conclusion is that any dissipative system is in fact passive when appropriate inputs and outputs are selected. We also derive an optimal stabilizing control result which can be considered as a general re-statement of Prigogine's Minimum Entropy Production principle (Glansdorff and Prigogine, 1971). In the light of these results, we finally discuss robustness issues in controller design, as it may become a relevant problem in the control of front or pulse-type spatial pattern formation.

The paper is organized as follows: In Section 2 we provide a general description of dissipative systems and their relevant properties. Connections with passivity are established in Section 3. In Sections 4 and 5 we develop the main results on optimal and robust stabilizing control of dissipative and discuss their implications in controller design. these ideas and results are illustrated, through the paper, on two examples involving complex reaction networks and a class of nonlinear diffusion-reaction dissipative system.

\section{Dissipative Systems: Description and Properties}

The class of systems we will consider in this work are derived from inventory balances of the form:

$$
\frac{d v}{d t}=\phi+\pi
$$

where $v$ is an $n$-dimensional vector of conserved properties (inventories), and $\phi$ and $\pi$ are 
the corresponding fluxes and production terms, respectively. Eqn (1) provides a macroscopic description of a process system which is associated to a given spatial domain $\mathcal{V}$ with smooth boundary $\mathcal{B}$. On this domain, the time-space evolution of the conserved properties can be obtained from (1) by introducing the density variables $z, f$ and $\Sigma$ so that:

$$
v=\int_{\mathcal{V}} z d \mathcal{V} \quad \pi=\int_{\mathcal{V}} \Sigma d \mathcal{V} \quad v=\int_{\mathcal{B}} f \mathbf{n} d \mathcal{B}
$$

where $\mathbf{n}$ is a unit vector pointing outwards of the boundary $\mathcal{B}$. Using these definitions and applying the divergence theorem over the inventories, the following set of partial differential evolution equations result:

$$
z_{t}+\partial_{k} f_{k}=\Sigma(z)+p
$$

where Einstein's notation is employed to denote spatial differentiation. The vector function $z\left(x_{k}, t\right) \in \mathcal{Z}$, defined over the set $\mathcal{D}=(\mathcal{V} \times \mathcal{B} \cup \mathcal{T})$ with $\mathcal{T}$ being the semi-open time interval $[0, \infty)$, will be referred to as the field. Vector functions $f_{k}, \Sigma(z)$ and $p$ describe microscopic fluxes through the domain, production densities and controls, respectively. Fluxes and production terms in dissipative systems have a particular structure, well motivated by thermodynamic arguments (see for instance Jou et al, 1996). In this way, the flux vector $f_{k}$ can be partitioned into convective and diffusive contributions as follows:

$$
f_{k}=\mathbf{v}_{k} z-f_{k}^{d}
$$

where $\mathbf{v}_{k}$ denotes the $k$ component of the fluid velocity field and $f_{k}^{d}$ represents the diffusive contribution. A formal characterization of the structure of fluxes in (2) is summarized next in the following assumptions:

Assumption A1. System (2) is equipped with a convex function a(z)

Assumption A2. The flux vector $f_{k}^{d}$ in (3) is related to the field $z$ through an expression of the form:

$$
f_{k}^{d}=L(A) X
$$

where $X=\partial_{k} A$, and $A$ is defined as the directional derivative of $a(z)$ so that $A=d_{z} a . L(A)$ in (4) is assumed to be positive definite and symmetric.

Assumptions A1 and A2, simply state the formal link existing between the densities of extensive variables $z$ (such as internal energy and mole numbers) and their intensive counterparts $A$ (temperature and chemical potentials). As discussed in Alonso and Ydstie (2001), such a connection is established through the existence of an entropy-like function (Assumption A1) which, by being convex, makes the map $z \rightarrow A$ one-to-one. In fact, $a(z)$ was employed by the authors to construct a convex function bounded from below which set up the basis to derive passive conditions. The process is as follows: given a stationary reference $z^{*}$, the new convex function $b\left(z ; z^{*}\right)$ is constructed as the difference between the original $a(z)$ and its supporting hyperplane at $z^{*}$ so that:

$$
b\left(z ; z^{*}\right)=a(z)-a\left(z^{*}\right)-\left(z-z^{*}\right)^{T} A^{*}
$$


As shown in Lemma 2 (Alonso and Ydstie, 2001), $b\left(z ; z^{*}\right)$ is bounded by the fields as:

$$
0 \leq q_{0}\left\|z-z^{*}\right\|_{2}^{2} \leq b\left(z ; z^{*}\right) \leq q_{1}\left\|z-z^{*}\right\|_{2}^{2}
$$

where $q_{0}$ and $q_{1}$ are strictly positive constants. It must be noted that since $b\left(z ; z^{*}\right)$ is itself convex, the map $\left(z-z^{*}\right) \rightarrow\left(A-A^{*}\right)$ is also one-to-one.

On the other hand, Eqn (4), in Assumption A2, imposes a relationship between diffusive fluxes and thermodynamic forces $X$ (the spatial gradients of $A$ ) known in irreversible thermodynamics as the Onsager-Casimir relationships (see for instance Jou et al, 1996 or Demirel, 2002).Finally, $\Sigma(z)$ in (2) is assumed to be Lipschitz continuous. Such a condition, is expressed next as an assumption:

Assumption A3. There exists a reference $z^{*}$ and a positive constant $\mu$ such that:

$$
\left(A-A^{*}\right)^{T}\left[\Sigma(z)-\Sigma\left(z^{*}\right)\right]+\ell_{\mu}\left(z ; z^{*}\right)=\mu\left(A-A^{*}\right)^{T}\left(A-A^{*}\right)
$$

with $\ell_{\mu}\left(z ; z^{*}\right) \geq 0$ for every $z$.

Note that condition (7) can always be imposed on $\Sigma(z)$ in terms of $A$, since it is Lipschitz and the map $\left(z-z^{*}\right) \rightarrow\left(A-A^{*}\right)$ is one-to-one. Consequently, there always exists a positive constant $\mu$ such that $\ell_{\mu}\left(z ; z^{*}\right) \geq 0$. The reason for writing the Lipschitz condition as in (7) is that it will allow us to easily state connections with time independent states operating both near and far from thermodynamic equilibrium (see Demirel, 2002). In this spirit, we include the following definition:

Definition 1. Pure Dissipative Systems are defined as those which, in addition to Assumptions A1-A2, satisfy that $\ell_{0}\left(z ; z^{*}\right)>0$ for every $z \neq z^{*}$ and $\ell_{0}\left(z^{*} ; z^{*}\right)=0$ in (7). If $\ell_{0}\left(z ; z^{*}\right) \geq 0$ only for $z$ such that $\left\|z-z^{*}\right\| \geq \varepsilon$ for some positive $\varepsilon$, the system will be Dissipative.

To illustrate the implications of Definition 1 on a dynamic context, let as consider the class of well-mixed systems (with states not distributed in space) on closed domains. In this case, equations (1) become of the form:

$$
\dot{z}=\Sigma(z)
$$

By choosing a reference $z^{*}$ and computing the time derivative of $b\left(z ; z^{*}\right)$ we have that:

$$
\dot{b}=\left(A-A^{*}\right)^{T}\left[\Sigma(z)-\Sigma\left(z^{*}\right)\right]=-\ell_{0}\left(z ; z^{*}\right)
$$

Since, from Eqn (6), $b$ is positive definite, we can explore the dynamic properties of the system with the help of LaSalle's theorem (see Khalil, 1996). To that purpose, let us define the set $\Omega=\left\{z \in \mathcal{Z} \mid\left\|z-z^{*}\right\| \geq \varepsilon\right\}$, where according to Definition $1, \dot{b} \leq 0$. Let $E \subset \Omega$ be the set of points where $\ell_{0}\left(z ; z^{*}\right)=0$, and $M$ the largest invariant set in $E$. Then, every solution in $\Omega$ approaches $M$ as $t \rightarrow \infty$. Note that for pure dissipative systems, the only element of the set $M$ is $z^{*}$, the equilibrium state. One important consequence of this argument, we will make use of later on in the sequel, is that the time integral of $\ell_{0}\left(z ; z^{*}\right)$, must be bounded as: 


$$
\int_{0}^{\infty} \ell_{0}\left(z ; z^{*}\right) d t<\infty
$$

Finally, the description of dissipative systems is completed with the appropriate boundary conditions. In that intention, we follow Alonso et al (2000) and partition the boundary $\mathcal{B}$ into three sets of positive measure $\mathcal{B}=\mathcal{B}_{c} \cup \mathcal{B}_{d} \cup \mathcal{B}_{0}$. $\mathcal{B}_{c}$ refers to that part of the boundary through which material flows with velocity $\mathbf{v}$. This boundary is divided into two disjoint sets satisfying:

$$
\begin{aligned}
\mathbf{v} \cdot \mathbf{n}\left(\mathcal{B}_{c}^{+}\right) & \geq 0 \\
\mathbf{v} \cdot \mathbf{n}\left(\mathcal{B}_{c}^{-}\right) & \leq 0
\end{aligned}
$$

with $\mathbf{n}$ being a unit vector normal to the surface and pointing outwards. Conditions (10) characterize the regions of the boundary where material leaves and enters, respectively. On the other hand, the sets $\mathcal{B}_{d} \cup \mathcal{B}_{0}$ define those parts of the boundary associated to diffusive fluxes and zero fluxes conditions. Boundary conditions on $\mathcal{B}$ are of the form:

$$
\begin{gathered}
z\left(\mathcal{B}_{c}^{-}\right)=z^{*} \\
\mathbf{f}^{d} \cdot \mathbf{n}\left(\mathcal{B}_{d}\right)=-H\left[A-A^{*}\right] \\
\mathbf{f}^{d} \cdot \mathbf{n}\left(\mathcal{B}_{0}\right)=0
\end{gathered}
$$

where $H$ is a positive definite matrix of transfer coefficients at the boundary and $z^{*}$ a given reference.

We end up this section with a typical example of dissipative systems, namely that of a complex reaction network. This example, taken from Gorban et al (2000), will serve to motivate Assumption A3 as well as to illustrate the definition of pure dissipative systems.

\subsection{Example 1: Dissipative Reaction Networks (Gorban et al, 2000)}

Let us consider an isolated and well-mixed (homogeneous) material system where $n$ chemical species (involving $p$ types of atoms) participate on a $r$-reaction network of the form:

$$
\sum_{i=1}^{n} \alpha_{i j} A_{i}=\sum_{i=1}^{n} \beta_{i j} A_{i} \quad \text { for } \quad j=1, \ldots, r
$$

$A_{i}$ represents the $i$-specie and $\alpha_{i j}$ and $\beta_{i j}$ are its corresponding stoichiometric coefficients for the $j$-reaction. Since the system is assumed to be isolated and well-mixed, the time evolution of the concentrations for the $n$ species can be described by a set of ordinary differential equations of the form:

$$
\dot{c}=\sum_{j=1}^{r} \nu_{j} W_{j}
$$

where $\nu_{j}$ are stoichiometric vectors having as elements $\nu_{i j}=\beta_{i j}-\alpha_{i j}$. The vector $c$ represents chemical specie concentrations and $W_{j}$ are the net reaction rates, which are assumed to obey the mass action law: 


$$
W_{j}=k_{j}^{+} \prod_{i=1}^{n} c_{i}^{\alpha_{i j}}-k_{j}^{-} \prod_{i=1}^{n} c_{i}^{\beta_{i j}}
$$

with $k_{j}^{+}$and $k_{j}^{-}$being positive kinetic parameters for the direct and inverse $j$-reaction rates, respectively. The phase space for this system is the space of positive concentrations constrained by the set of atomic conservation laws.

Next we show that system we just described is in fact Purely Dissipative (Definition 1) with respect to a constant reference $c^{*}$, defined as:

$$
W_{j}^{*}=0 \quad \text { for } \quad j=1, \ldots, r
$$

To that purpose, let us consider the convex function:

$$
a(c)=\sum_{i=1}^{n} c_{i}\left(\ln c_{i}-1\right)
$$

As discussed by Gorban et al (2000), this function is closely related to free energy for systems at constant temperature and volume. The dual $A$ to the field $c$ is obtained by computing the directional derivative of $a(c)$, being its elements $A_{i}=\ln c_{i}$ for $i=1, \ldots, n$. Function $b$-see Eqn (5)- in our example is now constructed as the difference between $a(c)$ and its supporting hyperplane at $c^{*}$ so that:

$$
b\left(c ; c^{*}\right)=a(c)-a\left(c^{*}\right)-A^{* T}\left(c-c^{*}\right)
$$

Substituting (15) (and the expression for $A$ ) into (16), and reordering terms, we get:

$$
b\left(c ; c^{*}\right)=\sum_{i=1}^{n} c_{i}\left[\ln \frac{c_{i}}{c_{i}^{*}}-1\right]+\sum_{i=1}^{n} c_{i}^{*}
$$

It is an easy matter to check whether $b\left(c ; c^{*}\right)$ is, in fact, positive for $c \neq c^{*}$ and $b\left(c^{*} ; c^{*}\right)=0$. Taking the time derivative of $b$ along (12) and using (7) with $\mu=0$ we have that:

$$
\begin{aligned}
\dot{b} & =\left(A-A^{*}\right)^{T}\left[\Sigma(z)-\Sigma\left(z^{*}\right)\right] \\
& =-\ell_{0}\left(c ; c^{*}\right)
\end{aligned}
$$

with

$$
\ell_{0}\left(c ; c^{*}\right)=-\left(A-A^{*}\right)^{T} \sum_{j=1}^{r} \nu_{j}\left(W_{j}-W_{j}^{*}\right)
$$

Note that $\ell_{0}\left(c^{*} ; c^{*}\right)=0$ so, in order to check whether this system is purely dissipative (Definition 1 ) we must show that $\ell_{0}\left(c ; c^{*}\right)>0$ for any $c \neq c^{*}$. To that purpose, let us define the following auxiliary variables: 


$$
\begin{gathered}
x_{j}=\prod_{i=1}^{n} c_{i}^{\alpha_{i j}} \quad y_{j}=\prod_{i=1}^{n} c_{i}^{\beta_{i j}} \\
z_{j}=\varphi_{j} x_{j} \quad \lambda_{j}=y_{j} z_{j}^{-1}
\end{gathered}
$$

for $j=1, \ldots, r$, and $\varphi_{j}=k_{j}^{+} / k_{j}^{-}$. Using (19) and (20) we can re-write (18) as:

$$
\begin{aligned}
\ell_{0}\left(c ; c^{*}\right)=\sum_{j=1}^{r} k_{j}^{-}\left(\bar{A}^{T} \nu_{j}\right) & \left(y_{j}-\varphi_{j} x_{j}\right)=\sum_{j=1}^{r} k_{j}^{-}\left[\ln \frac{y_{j}}{\varphi_{j} x_{j}}\right]\left(y_{j}-\varphi_{j} x_{j}\right) \\
\ell_{0}\left(c ; c^{*}\right) & =\sum_{j=1}^{r} k_{j}^{-} z_{j}\left(\lambda_{j}-1\right) \ln \lambda_{j} \\
& =\sum_{j=1}^{r} k_{j}^{-} z_{j} f\left(\lambda_{j}\right)
\end{aligned}
$$

with $f\left(\lambda_{j}\right)=\left(\lambda_{j}-1\right) \ln \lambda_{j}$. Since the phase space for the system is that of positive concentrations and $f\left(\lambda_{j}\right)$ is positive definite for every $\lambda_{j} \neq 1$, then it follows that $\ell_{0}\left(c ; c^{*}\right)>0$ except at $\lambda_{j}=1$ for all $j$. Recovering the original variables, $\lambda_{j}=1$ implies (for all $j$ ):

$$
k_{j}^{+} \prod_{i=1}^{n} c_{i}^{\alpha_{i j}}=k_{j}^{-} \prod_{i=1}^{n} c_{i}^{\beta_{i j}}
$$

which coincides with the reference (14).

\section{Passivity Conditions in the Context of Dissipative Systems}

In this section, we show that dissipative systems, as described by Eqn (2) and Assumptions A1-A3, are in fact passive in the standard sense of system's theory (Desoer and Vidyasagar, 1975), when appropriate inputs and outputs are selected. This result will set up the basis on which to derive connections with optimal control and asymptotic stabilization conditions.

Since the class of systems we are dealing with are in general distributed in time and space, let us first define the operator $\langle\alpha, \beta\rangle \mathcal{V}$ as that representing the inner product of two vector valued functions on the domain $\mathcal{V}$, so that:

$$
<\alpha, \beta>_{\mathcal{V}}=\int_{\mathcal{V}} \alpha^{T} \beta d V
$$

Using this operator, the $L_{2}$-norm of a given vector valued function $\alpha$ is defined as:

$$
\|\alpha\|_{\mathcal{V}}^{2}=<\alpha, \alpha>\mathcal{V}
$$


Lemma 1. Under assumptions A1-A3, there exists a function $B(t)$ bounded from below and satisfying:

$$
B(t+T)-B(t) \leq \int_{t}^{t+T}<y, u>_{\mathcal{V}} d s
$$

for any $t, T \geq 0$, with $y=A-A\left(z^{*}\right), u=p-p^{*}+\mu y$ and $z^{*}$ being a given stationary reference associated to a control $p^{*}$.

\section{Proof:}

Let us choose a stationary reference $z^{*}$ satisfying:

$$
\partial_{k} \mathbf{v}_{k} z^{*}=\partial_{k} f_{k}\left(A^{*}, X_{k}^{*}\right)+\Sigma\left(z^{*}\right)+p^{*}
$$

with boundary conditions of the form (10) and (11), and define the Kirchoff transform:

$$
\Gamma=\int_{A\left(z^{*}\right)}^{A(z)} L(A) d A
$$

Using the field in deviation form $\left(\bar{z}=z-z^{*}\right)$ and (23), system (2) can be re-written as:

$$
\bar{z}_{t}+\partial_{k} \mathbf{v}_{k} \bar{z}=\Delta \Gamma+\Sigma(z)-\Sigma\left(z^{*}\right)+p-p^{*}
$$

where $\Delta$ represents the usual Laplacian operator. Differentiating $b\left(z ; z^{*}\right)$-as defined in (5)- and combining it with (24) (see also Alonso et al, 2000) we get:

$$
\begin{aligned}
b_{t} & =\left[A-A\left(z^{*}\right)\right]^{T} \bar{z}_{t} \\
& =-\partial_{k}\left(\mathbf{v}_{k} b\right)+\bar{A}^{T} \Delta \Gamma+\bar{A}^{T}\left[\Sigma(z)-\Sigma\left(z^{*}\right)\right]+\bar{A}^{T}\left(p-p^{*}\right) \\
& =-\partial_{k}\left(\mathbf{v}_{k} b\right)+\bar{A}^{T} \Delta \Gamma-\ell_{\mu}\left(z ; z^{*}\right)+\mu \bar{A}^{T} \bar{A}+\bar{A}^{T}\left(p-p^{*}\right)
\end{aligned}
$$

where relation (7) in Assumption A3 has been employed. Using $y=A-A\left(z^{*}\right), u=p-p^{*}+\mu y$ and integrating over the spatial domain $\mathcal{V}$, we obtain:

$$
B_{t}=-\int_{\mathcal{B}_{c}} b\left(z ; z^{*}\right) \mathbf{v} \cdot \mathbf{n} d \mathcal{B}+<\bar{A}, \Delta \Gamma>_{\mathcal{V}}-L_{\mu}+<y, u>_{\mathcal{V}}
$$

with:

$$
\begin{aligned}
B & =\int_{\mathcal{V}} b\left(z ; z^{*}\right) d \mathcal{V} \\
L_{\mu} & =\int_{\mathcal{V}} \ell_{\mu}\left(z ; z^{*}\right) d \mathcal{V}
\end{aligned}
$$

Green's formula gives us:

$$
<\bar{A}, \Delta \Gamma>\mathcal{V}=\int_{\mathcal{B}_{d}} \bar{A}^{T} \mathbf{f}^{d} \cdot \mathbf{n} d \mathcal{B}-<\bar{X}, L(A) \bar{X}>\mathcal{V}
$$


which combined with (28) leads to:

$$
B_{t}=-\int_{\mathcal{B}_{c}} b\left(z ; z^{*}\right) \mathbf{v} \cdot \mathbf{n} d \mathcal{B}+\int_{\mathcal{B}_{d}} \bar{A}^{T} \mathbf{f}^{d} \cdot \mathbf{n} d \mathcal{B}-D_{\mu}+<y, u>\mathcal{V}
$$

with $D_{\mu}$ defined as:

$$
D_{\mu}=<\bar{X}, L(A) \bar{X}>\mathcal{V}+L_{\mu}
$$

Boundary conditions (10) and (11) make the first two terms at the right hand side of (30) negative. In addition, under Assumptions A1- A3 $D_{\mu} \geq 0$ for some positive $\mu$ and $B$ positive definite. Consequently, we can bound $B_{t}$ as:

$$
B_{t} \leq<y, u>\mathcal{V}
$$

and the result follows by integration over the interval $(t, t+T)$

For pure dissipative systems, the term $D_{\mu}$ in (31) with $\mu=0$ has a precise physical meaning: it quantifies the rate of entropy produced by the system on the domain. The function $D_{0}$ is positive definite for every $X \neq X^{*}, z \neq z^{*}$ and attains its minimum $-D_{0}=0$ - at the reference $\left(X^{*}, z^{*}\right)$. Thus for $p=p^{*}$, we have that $B_{t} \leq-D_{0}$ and the system evolves to the reference. This property is known in irreversible thermodynamics as the Principle of Minimum Entropy Production (Glansdorff and Prigogine, 1971). We will refer to $D_{\mu}$ as the Generalized Dissipation Function.

Corollary 1. Let $\int_{t}^{t+T}<\bar{A}, \bar{p}>_{\mathcal{V}} d s<\infty$ for every $t, T \geq 0$. Then $\bar{z}$ and $\bar{X}$ are bounded in the $L_{2}$-norm

\section{Proof:}

First we note that from Lemma 1, inequality (21) can also be written in terms of the generalized dissipation function (31) as:

$$
\begin{aligned}
B(t+T)-B(t) & \leq-\int_{t}^{t+T} D_{\mu} d s+\int_{t}^{t+T}<y, u>_{\mathcal{V}} d s \\
& \leq-\int_{t}^{t+T} L_{\mu} d s+\int_{t}^{t+T}<y, u>_{\mathcal{V}} d s
\end{aligned}
$$

For $\mu=0$ we have that

$$
\int_{t}^{t+T}<y, u>_{\mathcal{V}} d s=\int_{t}^{t+T}<\bar{A}, \bar{p}>_{\mathcal{V}} d s<\infty
$$

and $\int_{0}^{\infty} \ell_{0}\left(z ; z^{*}\right) d t<\infty$ (Eqn (9) in Assumption 3). Then, for every $t, T \geq 0, B(t)<\infty$. By integrating (6) on the domain $\mathcal{V}$, it follows that $q_{0}\|\bar{z}\|_{\mathcal{V}}^{2} \leq B$ so the field is bounded in the $L_{2}$-norm.

To show that this is also the case for $\bar{X}$ we note that, since $L(A)$ is positive definite (Assumption A2), there exists a positive constant $\delta_{1}$ such that: 


$$
\delta_{1}\|\bar{X}\|_{\mathcal{V}}^{2} \leq<\bar{X}, L(A) \bar{X}>\mathcal{V}
$$

Combining this inequality with (32) we have that:

$$
\delta_{1} \int_{t}^{t+T}\|\bar{X}\|_{\mathcal{V}}^{2} \leq B(t)-B(t+T)+\int_{t}^{t+T}\left(-L_{\mu}+<y, u>\mathcal{V}\right) d s
$$

and the result follows since the right hand side is bounded with $\mu=0$, for every $t, T>0$.

A number of consequences, useful for stabilization and robust control design, can be drawn from these results. These are summarized next in the form of remarks.

Remark 1. Systems satisfying Lemma 1 are is in fact passive in the sense given in standard system theory (see Desoer and Vidyasagar, 1975) with storage $B$, output $y$ and input $u$. Note that Pure Dissipative Systems are also passive for output $\bar{A}$ and input $\bar{p}$ since $D_{0} \geq 0$. For general dissipative systems there is always some positive $\mu$ such that $D_{\mu} \geq 0$. Thus any dissipative system can be rendered passive through proportional control $p-p^{*}=-\omega y+u^{\prime}$ with gain $\omega \geq \mu$. Such is the case since:

$$
\begin{aligned}
& <y, u>_{\mathcal{V}}=-(\omega-\mu)<y, y>_{\mathcal{V}}+<y, u^{\prime}>_{\mathcal{V}} \\
& <y, u>_{\mathcal{V}} \leq<y, u^{\prime}>_{\mathcal{V}}
\end{aligned}
$$

Remark 2. Corollary 1 guarantees that the field $z\left(x_{k}, t\right)$ is a member of $H^{1,2}\left(\mathcal{V} ; R^{n}\right)$. Such a condition was imposed by Alonso and Ydstie (2001) as an assumption in what they called the dissipation conditions. The present arguments state a direct connection with the physical basis of dissipative systems, formalized in Assumptions A1-A3. The main consequence of having $z\left(x_{k}, t\right) \in H^{1,2}\left(\mathcal{V} ; R^{n}\right)$ is that the field $\bar{z}$ (and any Lipschitz transformation map) can be expanded as an infinite series of the form:

$$
\bar{z}=\sum_{j=1}^{\infty} c_{j}(t) \phi_{j}(\mathbf{x})
$$

where the set $\left\{\phi_{i}\right\}_{i=1}^{\infty}$ represents a complete orthonormal basis satisfying the Euler-lagrange equations (Smoller, 1983):

$$
\Delta \phi_{j}=-\lambda_{j} \phi_{j}
$$

with appropriate boundary conditions. The eigenspectrum $\Lambda(\Delta)=\left\{\lambda_{j}\right\}_{j=1}^{\infty}$ consists of an ordered set of positive real numbers with the property that $\lambda_{i}<\lambda_{j}$ for every $i<j$. This property will be employed later on to derive asymptotic stabilization conditions. 


\section{Optimal and Robust Stabilization}

So far, we have shown that dissipative systems can be rendered passive by an appropriate selection of inputs and outputs. In the next two sections we go one step further and derive conditions for optimal and robust stabilizing control design. To that purpose, let us consider system (2) in deviation form with respect to a stationary reference $z^{*}$ satisfying (22), with boundary conditions of the form (10)-(11). Defining the field $\bar{z}=z-z^{*}$, the control $\bar{p}=p-p^{*}$, and using Kirchoff transform (23), system (2) becomes:

$$
\bar{z}_{t}+\partial_{k} \mathbf{v}_{k} \bar{z}=\Delta \Gamma\left(A ; A^{*}\right)+\left[\Sigma(z)-\Sigma\left(z^{*}\right)\right]+\bar{p}
$$

The following lemma summarizes a number of relations between the field and its dual, which will be employed in the sequel.

Lemma 2. Let $b\left(z ; z^{*}\right)$ be a convex function defined as in (5), then the following inequalities hold:

$$
\begin{aligned}
& \text { 1. }<\bar{A}, \bar{A}>_{\mathcal{V}} \geq \delta_{0}^{2}\|\bar{z}\|_{\mathcal{V}}^{2} \\
& \text { 2. }<\bar{X}, \bar{X}>_{\mathcal{V}} \geq \lambda_{1}\|\bar{A}\|_{\mathcal{V}}^{2} \\
& \text { 3. }<\bar{A}, \Delta \Gamma>_{\mathcal{V}} \leq-\lambda_{1} \delta_{1}\|\bar{A}\|_{\mathcal{V}}^{2}
\end{aligned}
$$

where $\delta_{0}$ and $\delta_{1}$ are the smallest eigenvalues of the $b$-Hessian and $L(A)$, respectively, over all possible values of the field, and $\lambda_{1}$ is the principal eigenvalue of the Laplacian operator.

\section{Proof:}

The first inequality is a direct consequence of Assumption A1. Since $b$ is convex, its Hessian, defined as:

$$
H=\frac{\partial^{2} b}{\partial \bar{z}_{i} \partial \bar{z}_{j}}
$$

is positive definite. Therefore, there exists a one-to-one map $\bar{A} \rightarrow \bar{z}$ so that that $\bar{A}=Q\left(z ; z^{*}\right) \bar{z}$ with $Q$ being a positive definite matrix (Dennis and Schnabel, 1983):

$$
Q\left(z ; z^{*}\right)=\int_{0}^{1} H\left[z^{*}+\varepsilon\left(z-z^{*}\right)\right] d \varepsilon
$$

By choosing $\delta_{0}$ as the smallest eigenvalue of $Q$ over all possible values of the field, inequality 1 follows.

To prove inequality 2 , we note that $\bar{z}$, and therefore its dual $\bar{A}$, are members of $H^{1,2}\left(\mathcal{V} ; R^{n}\right)$. Thus, we can expand each element of the field (and its dual) in terms of $\left\{\phi_{i}\right\}_{i=1}^{\infty}$ (Remark 2) so that:

$$
<\bar{A}, \bar{A}>\mathcal{V}=\sum_{j=1}^{\infty} a_{j}^{2}(t)
$$




$$
<\bar{A}, \Delta \bar{A}>\mathcal{V}=\sum_{j=1}^{\infty} a_{j}^{2}(t)<\phi_{j}(\mathbf{x}), \Delta \phi_{j}(\mathbf{x})>\mathcal{V}=-\sum_{j=1}^{\infty} \lambda_{j} a_{j}^{2}
$$

Since the eigenvalues $\lambda_{j}$ are positive real numbers satisfying that $\lambda_{i}<\lambda_{j}$ for every $i<j$, we can bound (37) as $\left\langle\bar{A}, \Delta \bar{A}>_{\mathcal{V}} \leq-\lambda_{1}\|\bar{A}\|_{\mathcal{V}}^{2}\right.$, where $\lambda_{1}$ is the principal eigenvalue (i.e. the smallest positive eigenvalue). In addition, $\|\bar{X}\|_{\mathcal{V}}^{2} \geq-<\bar{A}, \Delta \bar{A}>\mathcal{V}$ so by combining both inequalities we obtain:

$$
\|\bar{X}\|_{\mathcal{V}}^{2} \geq \lambda_{1}\|\bar{A}\|_{\mathcal{V}}^{2}
$$

In order to prove inequality 3, we make use of Green's formula (29) with boundary conditions (10)-(11) so that:

$$
\begin{aligned}
& <\bar{A}, \Delta \Gamma>_{\mathcal{V}}=\int_{\mathcal{B}_{d}} \bar{A}^{T} \mathbf{f}^{d} \cdot \mathbf{n} d \mathcal{B}-<\bar{X}, L(A) \bar{X}>_{\mathcal{V}} \\
& \leq-<\bar{X}, L(A) \bar{X}>_{\mathcal{V}} \leq-\delta_{1}\|\bar{X}\|_{\mathcal{V}}^{2}
\end{aligned}
$$

the result then follows by combining (38) and (40)

$$
<\bar{A}, \Delta \Gamma>_{\mathcal{V}} \leq-\delta_{1} \lambda_{1}\|\bar{A}\|_{\mathcal{V}}^{2}
$$

Next we connect dissipation and optimal stabilizing control by adapting Bellman-type sufficient conditions for optimality (Sepulchre et al, 1997) to our class of dynamic systems.

\section{Proposition 1.}

Consider a dissipative system (35), with a generalized dissipation function $D_{\mu}$ (31). Then, there exists a controller of the form $\bar{p}=-\omega \bar{A}$ with $\omega=2 \mu$ which makes the system exponentially stable and minimizes the functional:

$$
J=B\left(t_{f}\right)+\int_{0}^{t_{f}}\left[-<\bar{A}, \bar{f}>_{\mathcal{B}}+D_{\mu}+\frac{1}{4 \mu}<\bar{p}, \bar{p}>_{\mathcal{V}}\right] d t
$$

\section{Proof:}

As in Lemma 1, we start by computing the time derivative of $b$ along (35) and integrating over the domain, so that:

$$
B_{t}=-<\bar{X}, L(A) \bar{X}>_{\mathcal{V}}+<\bar{A}, \bar{f}>_{\mathcal{B}}+<\bar{A},\left[\Sigma(z)-\Sigma\left(z^{*}\right)\right]>_{\mathcal{V}}+<\bar{A}, \bar{p}>_{\mathcal{V}}
$$

Using (7) (Assumption A3) and the definition of $D_{\mu}$-Eqn (31)- Eqn (42) becomes:

$$
B_{t}=-D_{\mu}+<\bar{A}, \bar{f}>_{\mathcal{B}}+\mu<\bar{A}, \bar{A}>_{\mathcal{V}}+<\bar{A}, \bar{p}>_{\mathcal{V}}
$$


Boundary conditions (10)-(11) make $<\bar{A}, \bar{f}>_{\mathcal{B}} \leq 0$. Then, by applying the control law $\bar{p}=$ $-\omega \bar{A}$ we get:

$$
B_{t} \leq-D_{\mu}-\mu<\bar{A}, \bar{A}>_{\mathcal{V}}
$$

Note that the generalized dissipation function can be bounded as $D_{\mu} \geq<\bar{X}, L(A) \bar{X}>_{\mathcal{V}}$ (Assumption 3), so by Lemma $2 D_{\mu} \geq \delta_{1} \lambda_{1}\|\bar{A}\|_{\mathcal{V}}^{2}$ and therefore:

$$
B_{t} \leq-\left(\mu+\delta_{1} \lambda_{1}\right)\|\bar{A}\|_{\mathcal{V}}^{2}
$$

Using (6) and Lemma 2 we also have that

$$
\|\bar{A}\|_{\mathcal{V}}^{2} \geq \frac{\delta_{0}^{2}}{q_{1}} B
$$

Combining (44) and (45) we obtain $B_{t} \leq-\alpha B$, with $\alpha=\left(\mu+\delta_{1} \lambda_{1}\right) \delta_{0}^{2} q_{1}^{-1}>0$. Applying Gronwall lemma (see for instance Khalil, 1996) we then get $B(t) \leq B(0) \exp (-\alpha t)$ which implies that $\|\bar{z}\|_{\mathcal{V}}^{2} \rightarrow 0$ exponentially fast.

To prove the second part of the proposition (optimality), we first note that, since $B$ decreases and is bounded from below, the optimal control problem is well-defined. The optimal value attained can be computed by direct substitution of (43) into (41) so that:

$$
J^{*}=B\left(t_{f}\right)+\int_{0}^{t_{f}}\left[-B_{t}-\left(\mu-\frac{\omega^{2}}{4 \mu}\right)<\bar{A}, \bar{A}>_{\mathcal{V}}\right] d t=B(0)
$$

Finally, we show that in fact the control law $\bar{p}=-\omega \bar{A}$ is the optimal one. Suppose that the optimal control law is not $\bar{p}=-\omega \bar{A}$ but of the form:

$$
\bar{p}=v(t, \mathbf{x})-\omega \bar{A}
$$

Then:

$$
<\bar{p}, \bar{p}>_{\mathcal{V}}=<v, v>_{\mathcal{V}}+4 \mu^{2}<\bar{A}, \bar{A}>_{\mathcal{V}}-4 \mu<\bar{A}, v>_{\mathcal{V}}
$$

and

$$
<\bar{A}, \bar{p}>\mathcal{V}=<\bar{A}, v>\mathcal{V}-2 \mu<\bar{A}, \bar{A}>_{\mathcal{V}}
$$

so that Eqn (43) becomes:

$$
-<\bar{A}, \bar{f}>_{\mathcal{B}}+D_{\mu}=-B_{t}-\mu<\bar{A}, \bar{A}>_{\mathcal{V}}+<\bar{A}, v>_{\mathcal{V}}
$$

Substituting (46) and (47) into the functional we obtain: 


$$
\begin{aligned}
J= & B\left(t_{f}\right)+\int_{0}^{t_{f}}\left[-<\bar{A}, \bar{f}>_{\mathcal{B}}+D_{\mu}+\frac{1}{4 \mu}<\bar{p}, \bar{p}>_{\mathcal{V}}\right] d t \\
& B\left(t_{f}\right)+\int_{0}^{t_{f}}\left[-B_{t}+\frac{1}{4 \mu}<v, v>_{\mathcal{V}}\right] d t \\
= & B(0)+\frac{1}{4 \mu} \int_{0}^{t_{f}}<v, v>_{\mathcal{V}} d t \geq 0
\end{aligned}
$$

and the minimum is achieved only for $v=0$. Therefore $\bar{p}=-\omega \bar{A}$ is optimal

Relation (7), in Assumption A3, can be interpreted as a form of the Hamilton-Jacobi-Bellman equation (Sepulchre et al, 1997) while the objective functional (41) can be directly connected to the dissipation function (31). In this regard, Proposition 1 allows the design of feed-back control laws that minimizes the amount of entropy produced by the system. In particular, when applied to isolated Pure Dissipative Systems, Proposition 1 can be considered as an alternative statement of the Minimum Entropy Production Principle (Glansdorff and Prigogine, 1971). In this case, $\mu=0,<\bar{A}, \bar{f}>_{\mathcal{B}}=0$ (isolation) and the system spontaneously evolves with $\bar{p}=0$ so to minimize the amount of entropy produced.

\section{Robust Control of Dissipative Systems}

Let $S_{\infty}=\left\{\phi_{i}\right\}_{i=1}^{\infty}$ and $\Lambda(\Delta)=\left\{\lambda_{j}\right\}_{j=1}^{\infty}$ be the complete set of eigenfunctions and their corresponding eigenvalues, satisfying (34). We define two pair of disjoint sets $\left(S_{1}, \Lambda_{1}\right)$ and $\left(S_{2}, \Lambda_{2}\right)$, each of them containing a given number of eigenfunctions and their associated eigenvalues, and satisfying:

$$
S_{\infty}=S_{1} \cup S_{2} \quad \text { and } \quad \Lambda=\Lambda_{1} \cup \Lambda_{2}
$$

Let the number of elements in $S_{1}$ be finite (so $S_{2}$ contains the remaining -infinite- elements of the original set) and associate to $S_{1}, S_{2}$ the sub-fields $\bar{z}_{1}$ and $\bar{z}_{2}$, respectively, so that the field $\bar{z}$ can be partitioned as:

$$
\bar{z}=\bar{z}_{1}+\bar{z}_{2}=\sum_{j=1}^{\infty} c_{j}(t) \phi_{j}(\mathbf{x})
$$

Employing the sets $S_{1}$ and $S_{2}$ to partition the remaining terms in system (35), we can formally obtain the following equivalent representation:

$$
\begin{aligned}
& \left(\bar{z}_{1}\right)_{t}+\partial_{k} \mathbf{v}_{k} \bar{z}_{1}=\Delta \Gamma_{1}+\bar{\Sigma}_{1}\left(\bar{z}_{1}, \bar{z}_{2}\right)+\bar{p}_{1} \\
& \left(\bar{z}_{2}\right)_{t}+\partial_{k} \mathbf{v}_{k} \bar{z}_{2}=\Delta \Gamma_{2}+\bar{\Sigma}_{2}\left(\bar{z}_{1}, \bar{z}_{2}\right)+\bar{p}_{2}
\end{aligned}
$$

where, as in (48), we have that: 


$$
\begin{aligned}
\Gamma & =\Gamma_{1}+\Gamma_{2}=\sum_{j=1}^{\infty} \gamma_{j}(t) \phi_{j}(\mathbf{x}) \\
\bar{\Sigma} & =\bar{\Sigma}_{1}+\bar{\Sigma}_{2}=\sum_{j=1}^{\infty} \sigma_{j}(t) \phi_{j}(\mathbf{x}) \\
\bar{p} & =\bar{p}_{1}+\bar{p}_{2}=\sum_{j=1}^{\infty} \pi_{j}(t) \phi_{j}(\mathbf{x})
\end{aligned}
$$

A particular class of partition is that in which the set $S_{1}$ consists of a finite number of eigenfunctions, associated to the $n$ smallest eigenvalues. For dissipative systems, such partition allows the decomposition of the original distributed system into a slow (possibly unstable) and a fast and stable subsystem. This point can be easily justified in the light of the present theory. To see this, let us consider condition (7) and integrate the expression over the domain, so that:

$$
<\bar{A}, \bar{\Sigma}>_{\mathcal{V}}+L_{\mu}=\mu<\bar{A}, \bar{A}>_{\mathcal{V}}
$$

expanding $\bar{A}$ and $\bar{\Sigma}$ in terms of the set $S_{\infty}$, and using the orthonormality property of the eigenfunctions we also have that:

$$
\begin{aligned}
& <\bar{A}, \bar{\Sigma}>_{\mathcal{V}}=\sum_{j=1}^{\infty} a_{j} \sigma_{j} \\
& <\bar{A}, \bar{A}>_{\mathcal{V}}=\sum_{j=1}^{\infty} a_{j}^{2}
\end{aligned}
$$

substituting these expressions in (54) we obtain for a given $\mu>0$ :

$$
\sum_{j=1}^{\infty}\left(\mu a_{j}^{2}-a_{j} \sigma_{j}\right)=L_{\mu} \geq 0
$$

In order for this inequality to hold for any field satisfying (35) we need $\mu a_{j}^{2} \geq a_{j} \sigma_{j}$ for all $j$. This argument allows us to construct expressions equivalent to (54) for each subsystem (49) and (50). In particular we have for (50) that:

$$
<\bar{A}_{2}, \bar{\Sigma}_{2}>_{\mathcal{V}}+L_{\mu}=\mu<\bar{A}_{2}, \bar{A}_{2}>_{\mathcal{V}}
$$

To show that an open-loop stable subsystem (50) can always be found, we make use of the arguments employed in Proposition 1 (including Lemma 1): Define a $b$-function for $\bar{z}_{2}$ and compute its time derivative to obtain the equivalent of (42), which now becomes for $\bar{p}=0$ :

$$
\begin{aligned}
& B_{t} \leq-\left(\delta_{1} \lambda_{2}-\mu\right)\left\|\bar{A}_{2}\right\|_{\mathcal{V}}^{2} \\
& B_{t} \leq-\frac{\left(\delta_{1} \lambda_{2}-\mu\right) \delta_{0}^{2}}{q_{1}} B
\end{aligned}
$$


where $\lambda_{2}$ represents the smallest eigenvalue in $\Lambda_{2}$. For $\bar{z}_{2}$ to be exponentially stable all we have to do is to choose some $n$ large enough so that $\lambda_{2}>\mu \delta_{1}^{-1}$. The larger $n$, the faster will be the convergence $\left\|\bar{z}_{2}\right\|_{\mathcal{V}}^{2} \rightarrow 0$ of the stable subsystem. Such a decomposition property has been extensively used by Christofides and co-workers (see Chrsitofides (2001) for a general overview) to derive robust stabilizing nonlinear control schemes based on feed-back linearization of the slow subsystem.

Under condition (55), it is straightforward to apply previous results on passivity and optimal stabilizing control to a given partition (49) or (50). A possible control problem can be stated as follows:

Given a dissipative system (35), the objective is to ensure the stabilization of an arbitrary subsystem (50), while preserving the inherent dynamics of its complement (49):

$$
\left(\bar{z}_{1}\right)_{t}+\partial_{k} \mathbf{v}_{k} \bar{z}_{1}=\Delta \Gamma_{1}+\bar{\Sigma}_{1}\left(\bar{z}_{1}, 0\right)
$$

This problem is of relevance in the control of front and pulse pattern formation in distributed dissipative systems (see for instance, Shvartsman and Kevrekidis, 1998; Smagina et al, 2002). In this context, subsystem (56) describes the desired periodic pattern which becomes unstable by the influence of the complementary subsystem. Results on passivity and exponential stabilization can, in principle, be applied to this problem. However, the resulting control laws can produce extremely large actions due to the large $\mu$-bound required in (55). Alternative robust control schemes employed in finite dimensional robust nonlinear control (Khalil, 1996) can be easily adapted to deal with dissipative distributed systems as well. One of such robust stabilizing schemes is presented next.

\section{Proposition 2.}

Consider the dissipative system (35), let $\left(S_{2}, \Lambda_{2}\right)$ define a given set of arbitrary modes associated to a certain subfield $\bar{z}_{2}$, and $\bar{\Sigma}_{2}\left(\bar{z}_{1}, \bar{z}_{2}\right)$ be bounded as:

$$
\left\|\bar{\Sigma}_{2}\right\|_{\mathcal{V}} \leq \eta\left(\|\bar{A}\|_{\mathcal{V}}\right)\left\|\bar{A}_{2}\right\|_{\mathcal{V}}
$$

Then a control law:

$$
\begin{gathered}
\bar{p}=-\omega \bar{A}_{2}-\frac{\eta}{\left\|\bar{A}_{2}\right\|_{\mathcal{V}}} \bar{A}_{2} \quad \text { if } \quad \eta\left(\|\bar{A}\|_{\mathcal{V}}\right)\left\|\bar{A}_{2}\right\|_{\mathcal{V}} \geq \epsilon \\
\bar{p}=-\omega \bar{A}_{2}-\frac{\eta^{2}}{\epsilon} \bar{A}_{2} \quad \text { if } \quad \eta\left(\|\bar{A}\|_{\mathcal{V}}\right)\left\|\bar{A}_{2}\right\|_{\mathcal{V}}<\epsilon
\end{gathered}
$$

will make $\bar{z}_{2}$ to be ultimately bounded.

\section{Proof:}

As it is usual now, we start by defining a convex function $b\left(z_{2}, z_{2}^{*}\right)$, computing its time derivative along (35) and integrating over the domain to obtain, as in Proposition 1 (42):

$$
B_{t} \leq<\bar{A}_{2}, \Delta \Gamma_{2}>_{\mathcal{V}}+<\bar{A}_{2}, \bar{\Sigma}_{2}>_{\mathcal{V}}+<\bar{A}_{2}, \bar{p}_{2}>_{\mathcal{V}}
$$


Let $\lambda_{2}$ be the smallest eigenvalue in the set $\Lambda_{2}$, then Lemma 2 gives:

$$
B_{t} \leq-\lambda_{2} \delta_{1}\left\|\bar{A}_{1}\right\|_{\mathcal{V}}^{2}+<\bar{A}_{2}, \bar{\Sigma}_{2}>_{\mathcal{V}}+<\bar{A}_{2}, \bar{p}_{2}>_{\mathcal{V}}
$$

For $\eta\left(\|\bar{A}\|_{\mathcal{V}}\right)\left\|\bar{A}_{2}\right\|_{\mathcal{V}} \geq \epsilon$, we substitute (57) and the control into (58), so that:

$$
B_{t} \leq-\lambda_{2} \delta_{1}\left\|\bar{A}_{1}\right\|_{\mathcal{V}}^{2}+\eta\left(\|\bar{A}\|_{\mathcal{V}}\right)\left\|\bar{A}_{2}\right\|_{\mathcal{V}}-<\bar{A}_{2}, \bar{p}_{2}>_{\mathcal{V}}
$$

and

$$
B_{t} \leq-\frac{\left(\lambda_{2} \delta_{1}+\omega\right) \delta_{0}^{2}}{q_{1}} B
$$

so that, whenever $\eta\left(\|\bar{A}\|_{\mathcal{V}}\right)\left\|\bar{A}_{2}\right\|_{\mathcal{V}} \geq \epsilon, B$ (and therefore the field) will evolve as:

$$
B(t) \leq B(0) \exp (-\alpha t)
$$

with $\alpha=\left(\lambda_{2} \delta_{1}+\omega\right) \delta_{0}^{2} q_{1}^{-1}$ being a configurable parameter that can be made arbitrarily large by increasing the gain $\omega$.

When $\eta\left(\|\bar{A}\|_{\mathcal{V}}\right)\left\|\bar{A}_{2}\right\|_{\mathcal{V}}<\epsilon$ we have:

$$
\begin{gathered}
B_{t} \leq-\left(\lambda_{2} \delta_{1}+\omega\right)\left\|\bar{A}_{2}\right\|_{\mathcal{V}}^{2}+\eta\left\|\bar{A}_{2}\right\|_{\mathcal{V}}-\frac{\eta^{2}\left\|\bar{A}_{2}\right\|_{\mathcal{V}}^{2}}{\epsilon} \\
B_{t}+\alpha B \leq \psi-\frac{\psi^{2}}{\epsilon}
\end{gathered}
$$

with $\psi=\eta\left\|\bar{A}_{2}\right\|_{\mathcal{V}}$. The right hand term of the inequality is only positive in the interval $\psi \in(0, \epsilon)$ and attains its maximum at $\psi=\epsilon / 2$. Solution of inequality (59) gives:

$$
B(t) \leq B(0) \exp (-\alpha t)+\frac{\epsilon}{4 \alpha}[1-\exp (-\alpha t)]
$$

In the limit as $t \rightarrow \infty$, a bound for $B$ (and therefore the field) is found:

$$
\lim _{t \rightarrow \infty} B=\frac{\epsilon}{4 \alpha}
$$

which implies that the field is ultimately bounded.

\subsection{Example: Robust Modal Control of the Fitzhugh-Nagumo System}

The ideas presented so far on passivity and modal stabilization will be illustrated on a class of dissipative system known as Fitzhugh-Nagumo (Murray, 1993). This system can be considered as a simplified version of the Hodgkin-Huxley model (Hodgkin and Huxley, 1952) derived 
to explain nerve-impulse propagation. It is described by a pair of coupled reaction-diffusion equations of the form:

$$
\begin{aligned}
& v_{t}=k \Delta v+f(v)-w+p^{\prime} \\
& w_{t}=\delta k \Delta w+\varepsilon\left(v-\gamma_{1} w\right)
\end{aligned}
$$

with $f(v)=v-v^{3}$. The system is defined on a rectangular domain $\mathcal{V} \in(-1 \leq x \leq 1,-1 \leq$ $y \leq 1)$ with zero-flux conditions at the boundary. The fields $v$ and $w$ correspond to the concentrations of activator and inhibitor species, respectively, and $p^{\prime}$ represents the actuator function to be manipulated through control. Parameters $\delta$ and $\varepsilon$ in Eqn (61) are the ratio of diffusivities and reaction rates for the two species. The interaction between diffusion and nonlinear reaction in this system induces a rich variety of stationary, as well as oscillatory, spatial patterns, usually characterized by relatively sharp concentration fronts (Shvartsman and Kevrekidis, 1998). One of such patterns can be seen in Figure 1.

To show that the system is dissipative with respect to the homogeneous stationary reference $v^{*}=w^{*}=0$, we choose a simple quadratic function:

$$
b=\frac{1}{2}\left(\varepsilon v^{2}+w^{2}\right)
$$

compute $\bar{A}$, which now becomes $\bar{A}=[\varepsilon v, w]^{T}$, and determine $\ell_{\mu}$ from relation (7). By direct substitution we get:

$$
\ell_{\mu}(v, w)=\varepsilon v^{2}\left(\mu \varepsilon-1+v^{2}\right)+\left(\mu+\varepsilon \gamma_{1}\right) w^{2}
$$

which is positive definite for $\mu>\varepsilon^{-1}$. Consequently the system is dissipative according to Definition 1. However, the system is not purely dissipative since $\ell_{0}$ is negative on the interval:

$$
v \times w \rightarrow(-1,1) \times\left(-|v| \sqrt{\frac{1-v^{2}}{\gamma_{1}}},|v| \sqrt{\frac{1-v^{2}}{\gamma_{1}}}\right)
$$

Since the system is dissipative, we can use Lemma 1 (and Remark 1) to enforce passivity. In our case this can be done by choosing an input-output pair $(u, y)$ of the form:

$$
\begin{aligned}
& u=p^{\prime}+\mu y \\
& y=\varepsilon v
\end{aligned}
$$

\section{Robust Modal Control}

Finally, we illustrate on the the Fitzhugh-Nagumo system (60)-(61) the application of Proposition 1. The objective here is to ensure the stabilization of a given arbitrary sub-field $\left(v_{2}, w_{2}\right)$, associated to a spectral pair $\left(S_{2}, \Lambda_{2}\right)$, while preserving the inherent dynamics of its complement $\left(v_{1}, w_{1}\right)\left(S_{1}, \Lambda_{1}\right)$. In this example, we assume that $f(v)$ is unknown but satisfies an inequality 
of the form (57). The sub-field $\left(v_{1}, w_{1}\right)$ we want to preserve consists of 8 modes, picked up among the 50 slowest modes of the system. Under condition $v_{2}=0 ; w_{2}=0$, everywhere in $\mathcal{V}$, the modes associated to the sub-fields $\left(v_{1}, w_{1}\right)$ oscillate and describe a stable limit cycle. The oscillatory response for the 8 modes is presented in Figure 2. The effect of such behavior is a periodic pattern as the one depicted in Figure 3.

In order to apply Proposition 1, we define a new input $p=p^{\prime}-w$ and construct the control law as:

$$
p=\left\{\begin{array}{ccc}
-\omega v_{2}-\eta\left(\|v\|_{V}\right) \frac{v_{2}}{\|v\|_{V}} & \text { if } & \eta\left(\|v\|_{V}\right)\left\|v_{2}\right\|_{V} \geq \epsilon \\
-\omega v_{2}-\eta^{2}\left(\|v\|_{V}\right) \frac{v_{2}}{\epsilon} & \text { if } & \eta\left(\|v\|_{V}\right)\left\|v_{2}\right\|_{V}<\epsilon
\end{array}\right\},
$$

The implementation of control law (62) requires measurements of the field $(v, w)$. With these measurements, the sub-field $v_{2}$ is re-produced by discounting the modes we want to preserve. If $n$ is the number of elements in the set $S_{1}=\left\{\phi_{j}^{1}\right\}_{j=1}^{n}$, reconstruction proceeds as follows:

$$
v_{2}=v-\sum_{j=1}^{n}<v, \phi_{j}^{1}>_{\mathcal{V}} \phi_{j}^{1}
$$

The effect of control law (62) with $\omega=0.1 ; \epsilon=0.001$, on the norm $\left\|v_{2}\right\|_{\mathcal{V}}$ is presented in Figure 4, showing that after a small transient the sub-field remains bounded. The evolution of some of the modes (associated to the sub-field $v_{2}$ ) under control as compared with the same modes in open loop in depicted in Figure 5. Figure 6 represents the evolution of the first 10 slowest modes associated to the field $v$. Note that some modes $(1,2,5$ and 10) oscillate thus demonstrating that the control law does, in fact, preserve the dynamics of the modes associated to the sub-field $v_{1}$

\section{Conclusions}

In this work, we explore new links between the underlying physics of dissipative process systems and nonlinear control. The existence of an entropy-like function allows us to relate dissipation with a Hamilton-Jacobi-Bellman type equation. Such relation is employed to state passivity conditions for dissipative systems. In this way, one main conclusion is that any dissipative system can be rendered passive by appropriate selection of inputs and outputs. We also connect dissipation with optimal stabilizing control and use this result to re-state the principle of Minimum Entropy Production. Finally, Robustness issues in controller design, are discussed in the context of front or pulse spatial pattern stabilization.

\section{Acknowledgements}

The authors acknowledge generous support from Plan Nacional de Investigación Científica (MCyT-Spain), project PPQ2001-3643 


\section{$7 \quad$ References}

Alonso, A.A. and B.E. Ydstie (1996) Process Systems, Passivity and the Second Law of Thermodynamics. Comp. \& Chem. Eng., 20: S1119

Alonso, A.A., Banga, J.R. and I. Sanchez (2000) Distributed Process Systems: Theory and Applications. AIChE J. 46(8):1593

Alonso, A.A. and B.E. Ydstie (2001) Stabilization of Distributed Process Systems Using Irreversible Thermodynamics. Automatica, 37:1739

Balas, M.J. (1983) The galerkin Method and Feedback Control of Linear Distributed Parameter Systems. J. Math. Anal. Appl. 91: 527

Christofides, P.D. and P. Daoutidis (1996) Nonlinear Control of Diffusion-Convection-Reaction Processes. Comp. Chem. Eng. 20(s): 1071.

Christofides, P.D. and P. Daoutidis (1997a) Control of Nonlinear Distributed Parameter Processes: Recent Results and Future Research Directions. Chemical Process Control IV, AIChE Symposium Series. 93: 302

Christofides, P.D.and P. Daoutidis (1997b) Finite-Dimensional Control of Parabolic PDE Systems Using Approximate Inertial Manifolds. J. Math. Anal. Appl. 216: 398

Christofides, P.D. (2001) Nonlinear and Robust Control of PDE Systems. Birhauser, Boston

Demirel, Y (2002) Nonequilibrium Thermodynamics. Elsevier, Amsterdam

Dennis, J.R. and J.E. Schnabel (1983) Numerical Methods for Unconstrained Optimization and Nonlinear Equations. Prentice Hall, Englewood Cliffs, N.J.

Desoer, C.A., and M. Vidyasagar (1975) Feedback Systems: Input-Output Properties. Academic Press, New York

Dochain, D., Babary, J.P. and M.N. Tali-Manaar (1992) Modelling and Adaptive Control of Nonlinear Distributed Parameter Bioreactors by Orthogonal Collocation. Automatica, 68: 873.

Glansdorff, P. and I. Prigogine (1971) Thermodynamic Theory of Structure, Stability and Fluctuations. Wiley, New York.

Gorban, A.N., Karlin, I.V., Zmievskii, V.B. and S.V. Dymova (2000). Reduced Description in Reaction Kinetics. Physica A, 275 (3-4): 361

Hangos, K.M., Alonso, A.A., Perkins, J.D. and B.E. Ydstie (1999) Thermodynamic Approach to Structural Stability of Process Plants. AIChE J. 45: 802. 
Hodgkin A.L. and A. F. Huxley (1952) A quantitative description of membrane current and its application to conduction and excitation in nerve. J. Physiol., 117: 500.

Jou, D., Casas-Vazquez, G. Lebon (1996) Extended Irreversible Thermodynamics. SpringerVerlag, New York

Khalil, H.K. (1996) Nonlinear Systems. Prentice Hall, Englewood Cliffs, N.J.

Lasiecka, I. (1995) Control of Systems Governed by Partial Differential Equations: A Historical Perspective. Proceedings of 34th IEEE Conference on Decision and Control, 2792, New Orleans, LA

Murray, J.D. (1993) Mathematical Biology. Springer, New York

Sepulchre, R., M. Jankovic and P. Kokotovic (1997) Constructive Nonlinear Control. SpringerVerlag, London

Shvartsman, S.Y. and I.G. Kevrekidis (1998) Nonlinear Model Reduction for Control of Distributed Systems: a Computer Assisted Study. AIChE J. 44(7): 1579

Smagina, Y., Nekhamkina, O. and M. Sheintuch (2002) Stabilization of Fronts in a ReactionDiffusion System: Aplication of the Gershgorin Theorem. Ind. Eng. Chem. Res. 41: 2023

Smoller, J. (1983) Shock Waves and Reaction-Diffusion Equations. Springer-Verlag, New York

Ydstie, B.E., A.A. Alonso (1997) Process Systems and Passivity Via the Clausius-Plank Inequality. Syst. \& Cont. Letters 30: 253

Ydstie, B.E. (2002) Passivity Based Control Via the Second Law. Comp. \& Chem. Eng. 26: 1037 


\section{List of Figures}

Figure 1. A typical spatial pattern produced by the Fitzhugh-Nagumo system, with parameters $k=10^{-4}, \delta=2.5, \varepsilon=0.03, \gamma_{1}=2$. (1a) Snapshot corresponding to the $v$ field. (1b) Snapshot corresponding to the $w$ field.

Figure 2. Periodic response exhibited by the active modes associated to $v_{1}$ under condition $v_{2}=0$ and $w_{2}=0$, everywhere in $\mathcal{V}$.

Figure 3. Spatial pattern produced under condition $v_{2}=0$ and $w_{2}=0$, everywhere in $\mathcal{V}$. (2a) Snapshot corresponding to the $v$ field. (2b) Snapshot corresponding to the $w$ field.

Figure 4. Transient evolution of the norm $\left\|v_{2}\right\|_{\mathcal{V}}$ under control law (62). The control parameters employed were $\omega=0.1$ and $\epsilon=0.001$.

Figure 5. A comparison between open-loop and closed loop modal evolution for some modes associated to $v_{2}$. Plots at the left represent the open loop behavior. Plots at the right represent the evolution under control.

Figure 6. Evolution of the first 10 slowest modes associated to the field $v$. Modes in oscillation are $1,2,5$ and 10 and belong to the sub-field $v_{1}$ 
Dissipative systems: from physics to robust nonlinear control.

INTERNATIONAL JOURNAL OF ROBUST AND NONLINEAR CONTROL, 14(2), 157-179

Fig. 1
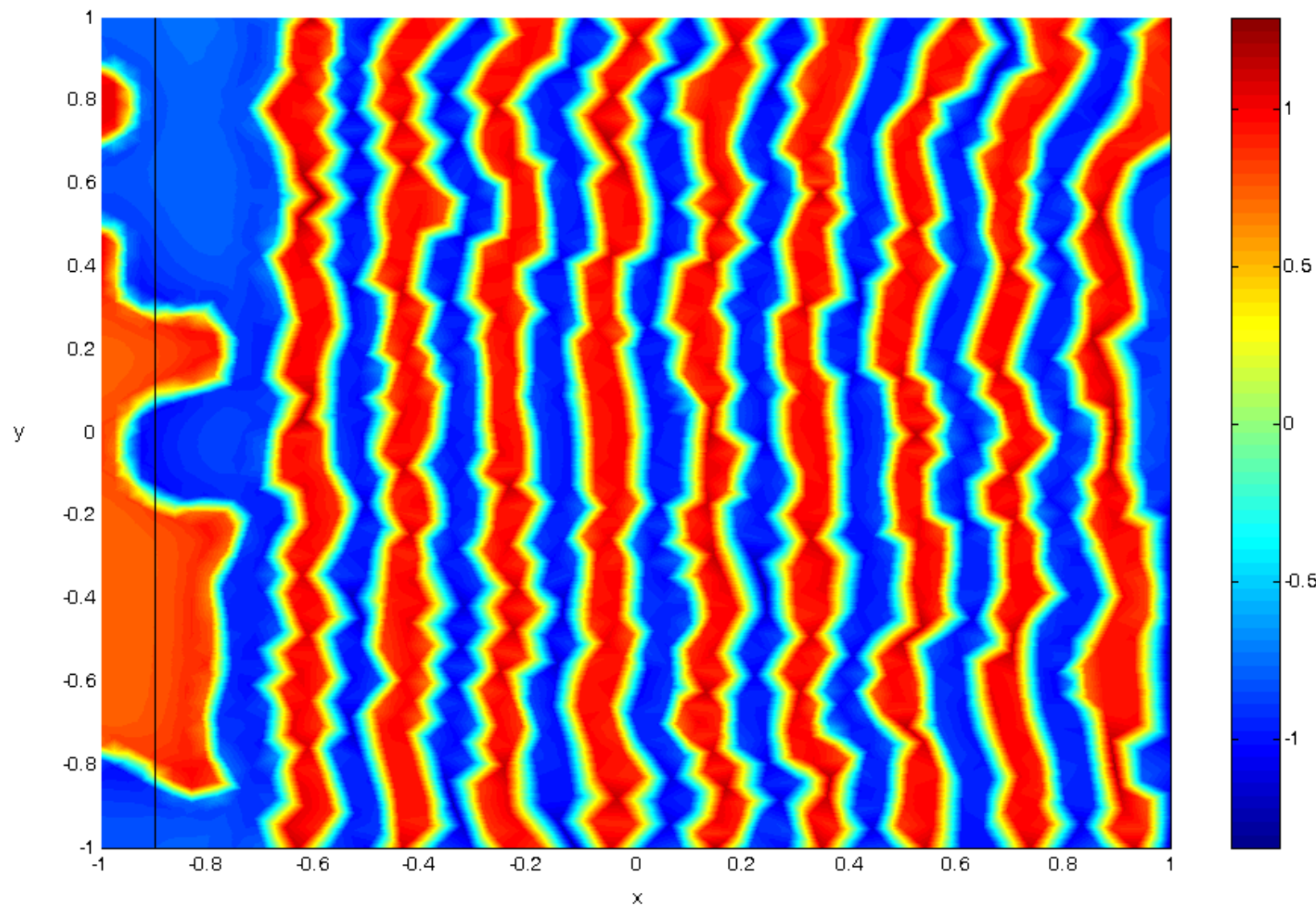

(1a)
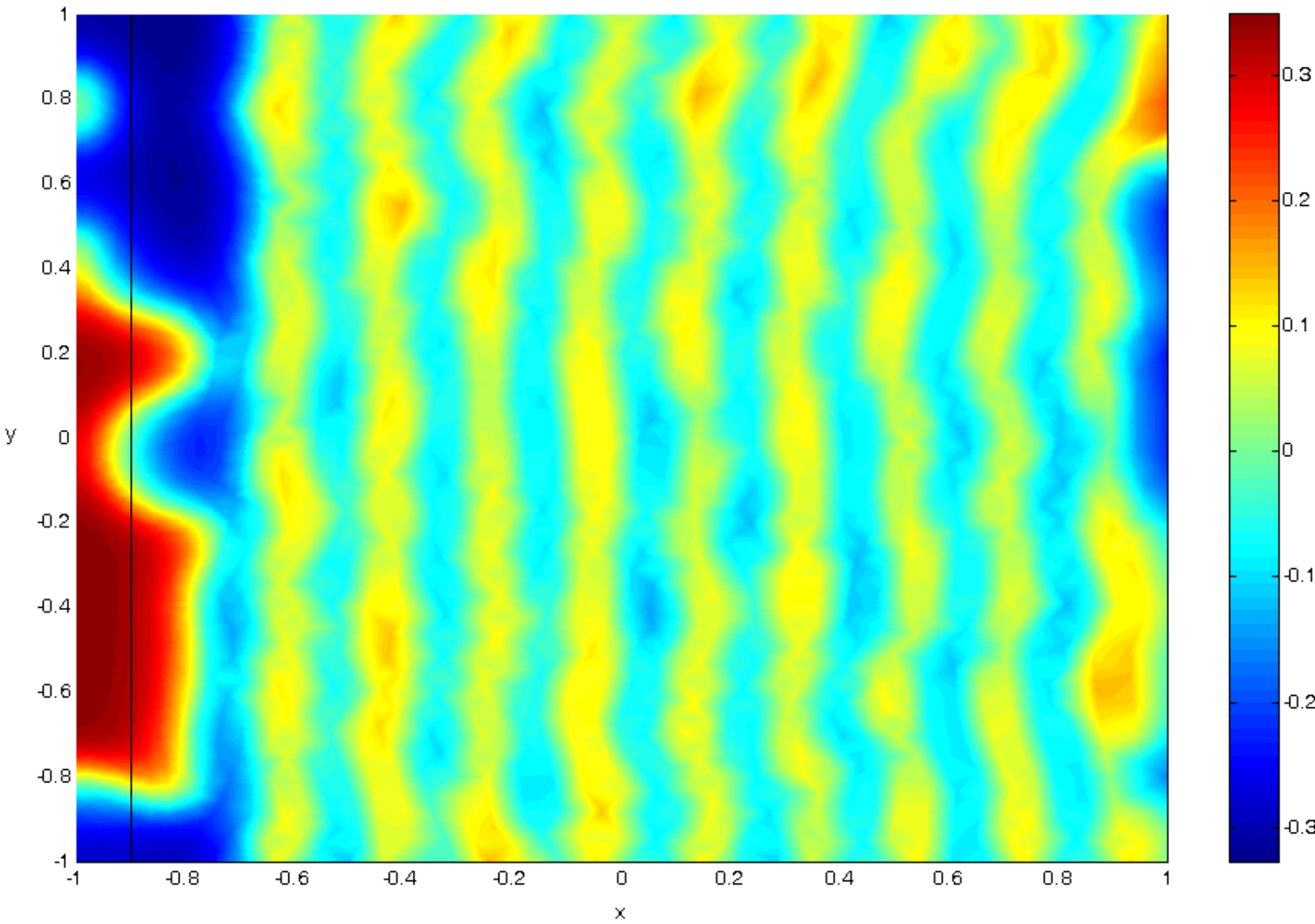

(1b) 
Fig. 2

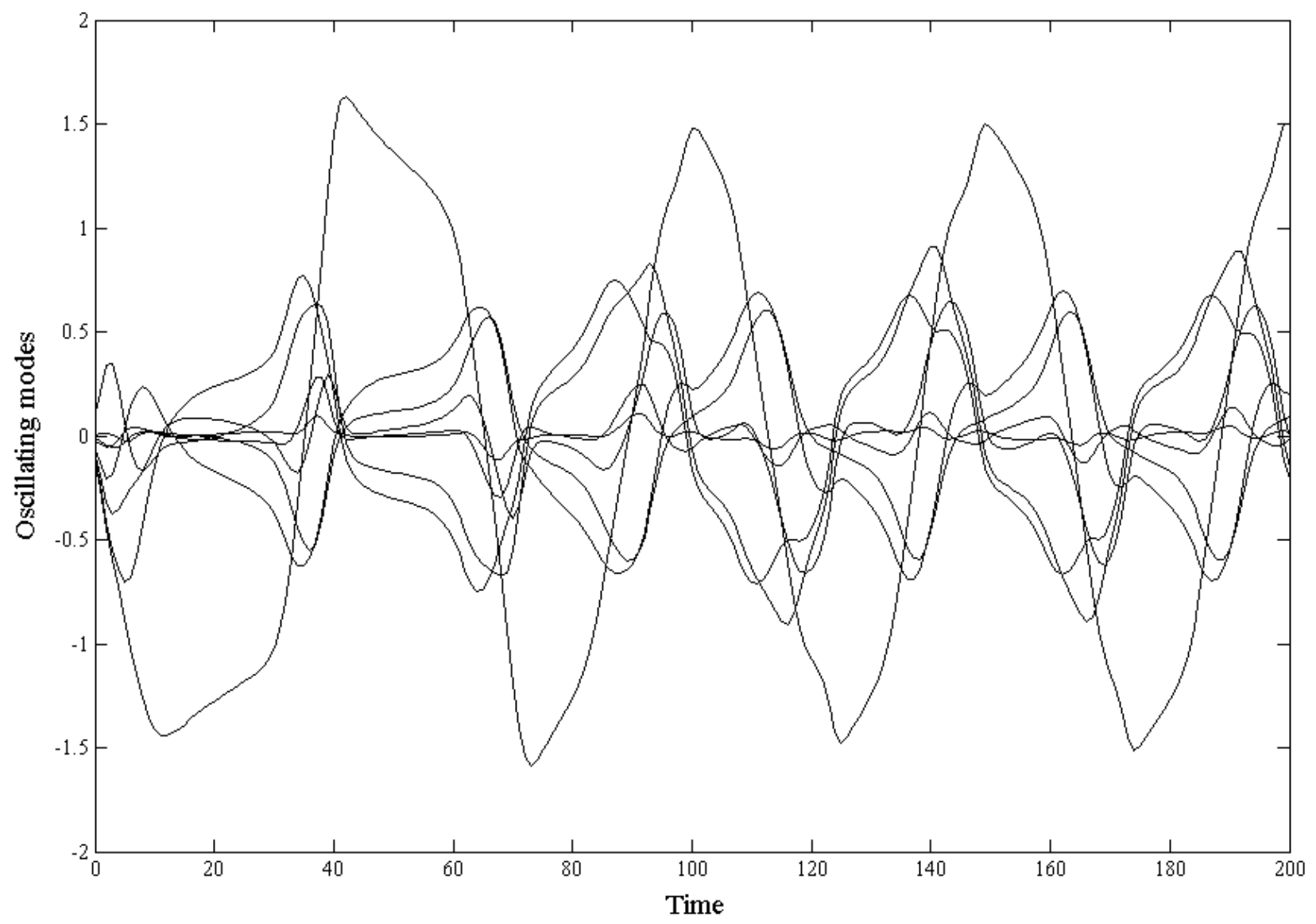


Alonso, A. A., Fernandez, C. V., Banga, J. R. (2004)

Dissipative systems: from physics to robust nonlinear control.

INTERNATIONAL JOURNAL OF ROBUST AND NONLINEAR CONTROL, 14(2), 157-179 Fig. 3
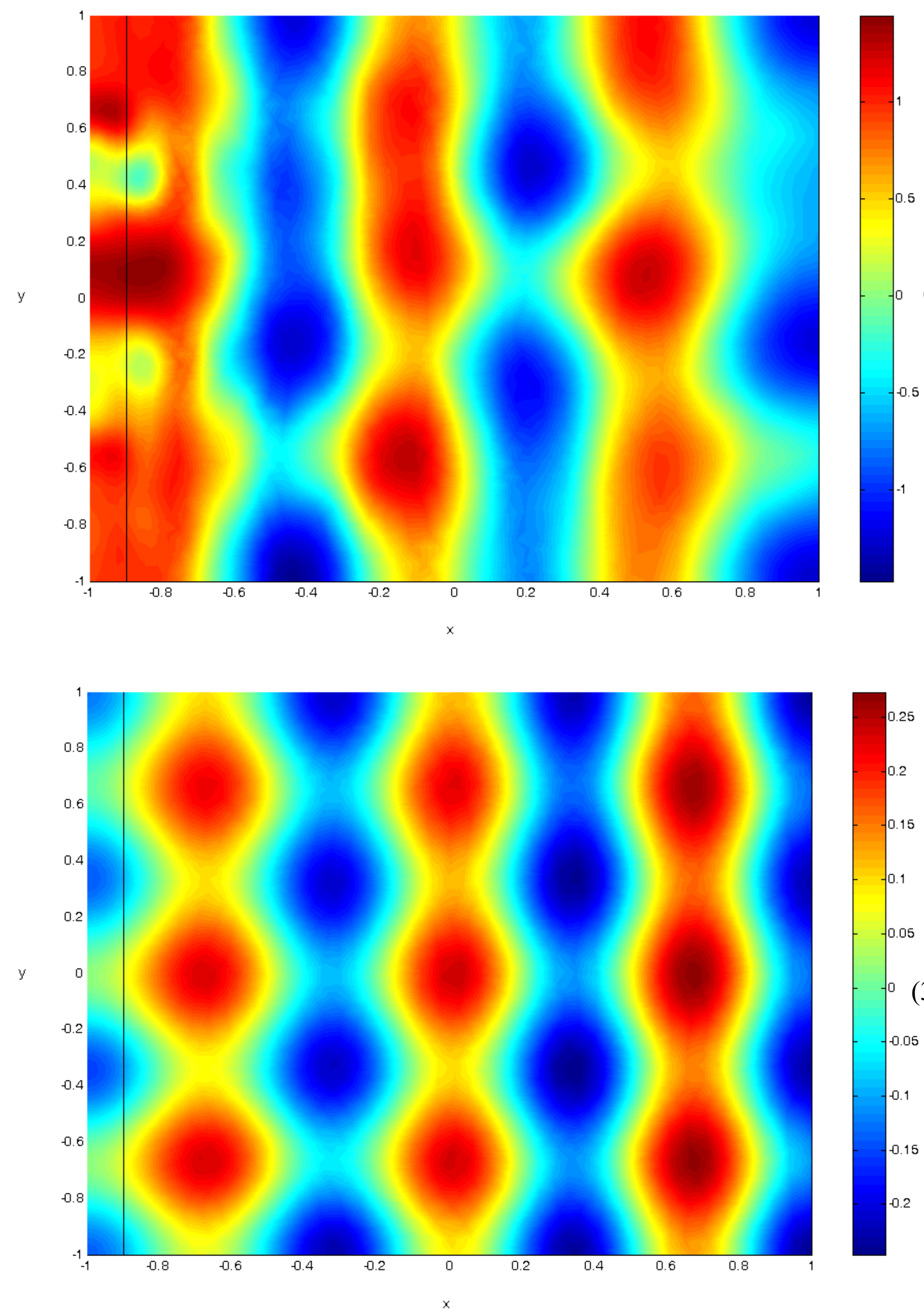
To cite this article:

Alonso, A. A., Fernandez, C. V., Banga, J. R. (2004)

Dissipative systems: from physics to robust nonlinear control.

INTERNATIONAL JOURNAL OF ROBUST AND NONLINEAR CONTROL, 14(2), 157-179

Fig. 4

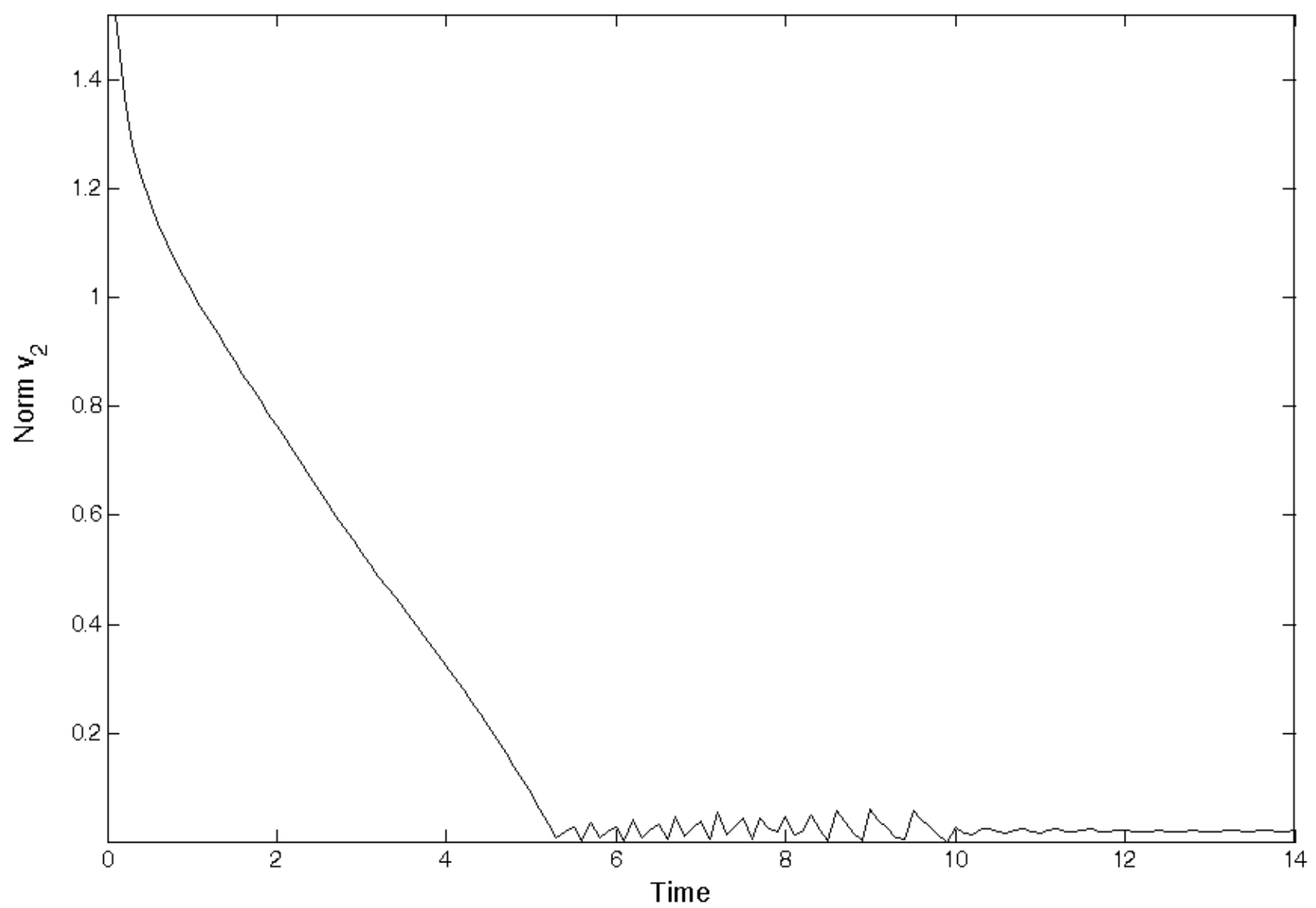


Fig. 5
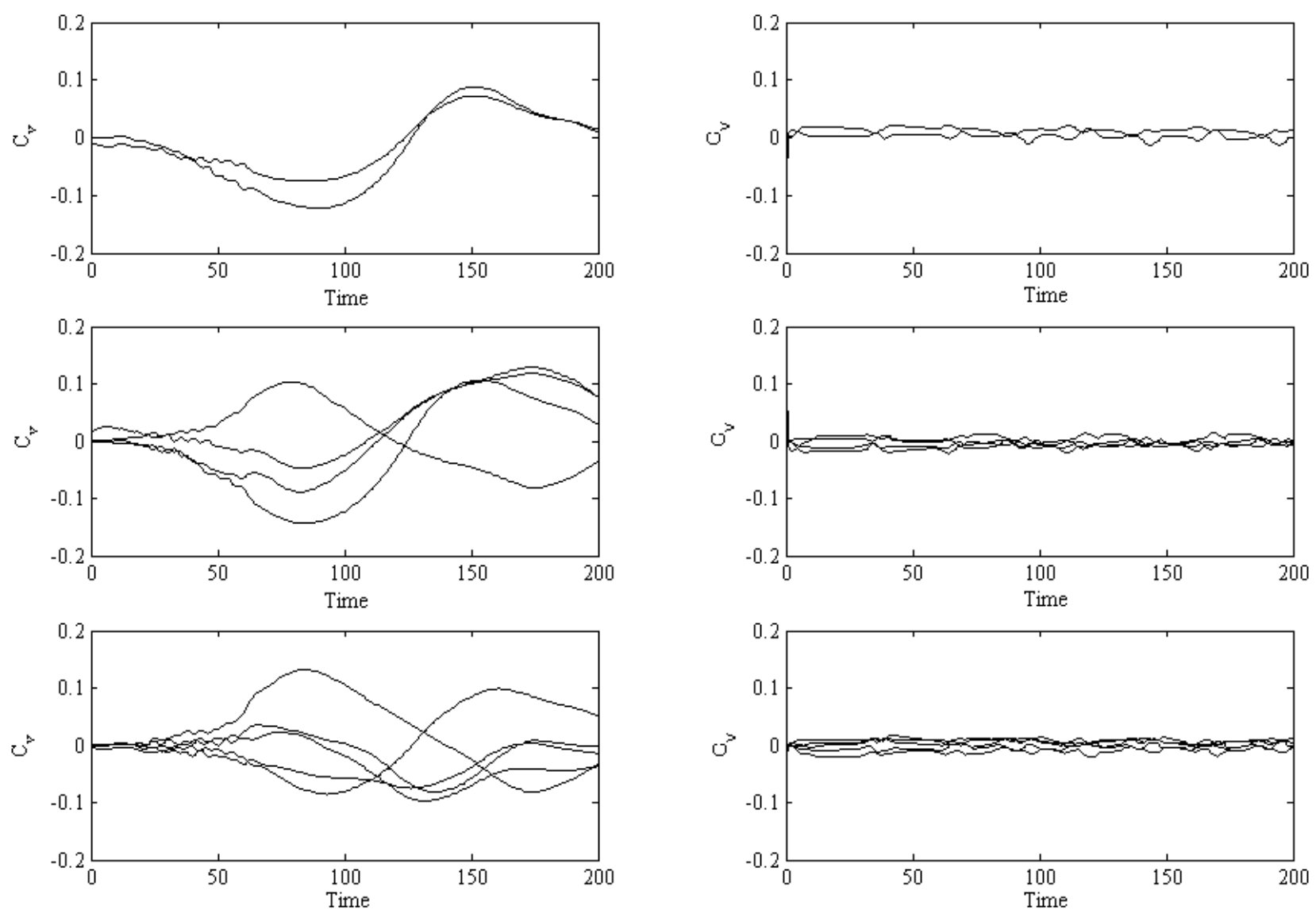
To cite this article:

Alonso, A. A., Fernandez, C. V., Banga, J. R. (2004)

Dissipative systems: from physics to robust nonlinear control.

INTERNATIONAL JOURNAL OF ROBUST AND NONLINEAR CONTROL, 14(2), 157-179

Fig. 6

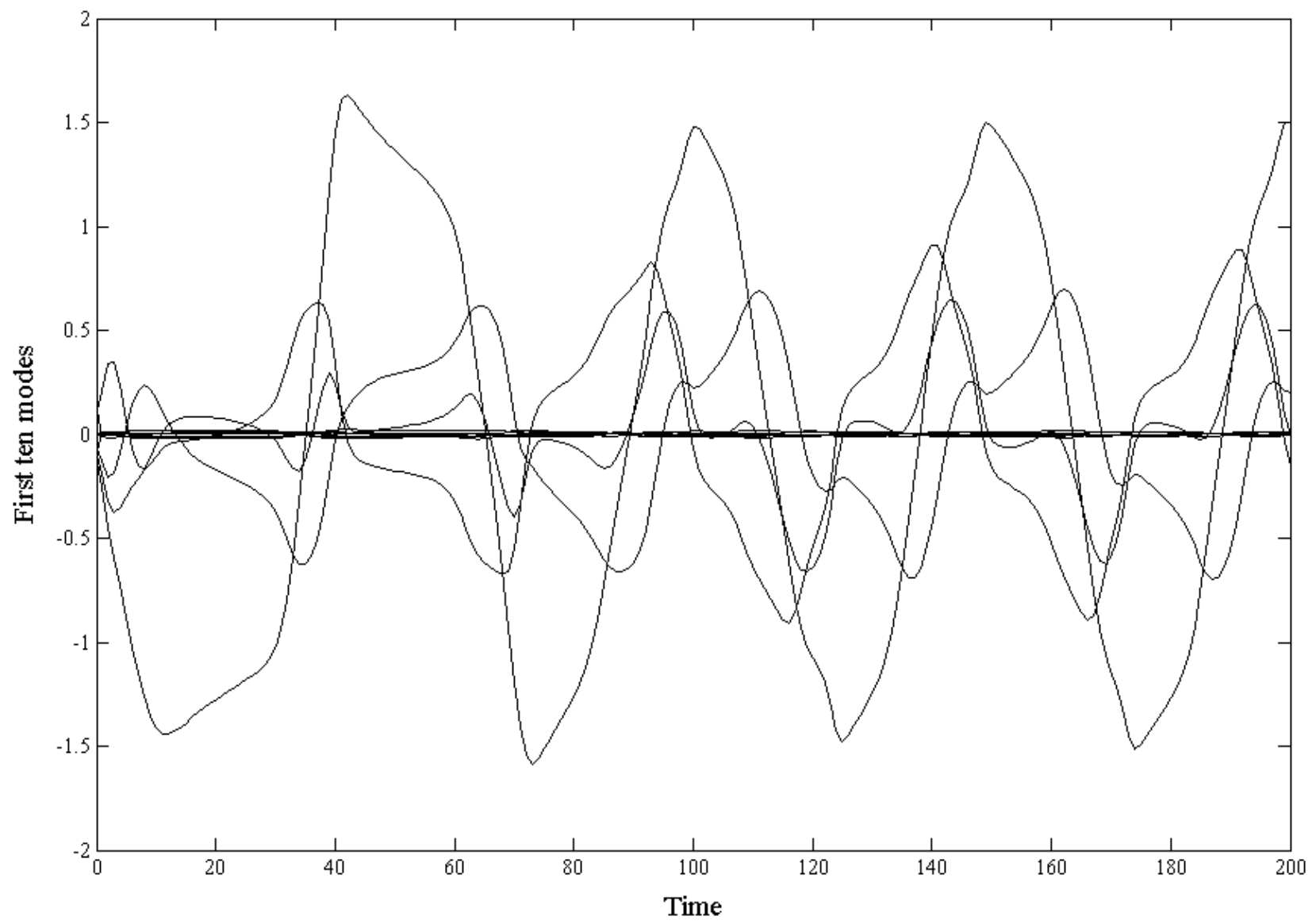

